\title{
Nonlinear Pulse Shaping in Fibres for Pulse Generation and Optical Processing
}

\author{
Sonia Boscolo ${ }^{1}$ and Christophe Finot ${ }^{2}$ \\ ${ }^{1}$ Photonics Research Group, School of Engineering and Applied Science, Aston University, Birmingham B4 7ET, UK \\ ${ }^{2}$ Laboratoire Interdisciplinaire Carnot de Bourgogne, UMR 6303 CNRS, Université de Bourgogne, 21078 Dijon, France
}

Correspondence should be addressed to Sonia Boscolo, s.a.boscolo@aston.ac.uk

Received 16 November 2011; Accepted 9 January 2012

Academic Editor: Juan Diego Ania Castañón

Copyright (C) 2012 S. Boscolo and C. Finot. This is an open access article distributed under the Creative Commons Attribution License, which permits unrestricted use, distribution, and reproduction in any medium, provided the original work is properly cited.

\begin{abstract}
The development of new all-optical technologies for data processing and signal manipulation is a field of growing importance with a strong potential for numerous applications in diverse areas of modern science. Nonlinear phenomena occurring in optical fibres have many attractive features and great, but not yet fully explored, potential in signal processing. Here, we review recent progress on the use of fibre nonlinearities for the generation and shaping of optical pulses and on the applications of advanced pulse shapes in all-optical signal processing. Amongst other topics, we will discuss ultrahigh repetition rate pulse sources, the generation of parabolic shaped pulses in active and passive fibres, the generation of pulses with triangular temporal profiles, and coherent supercontinuum sources. The signal processing applications will span optical regeneration, linear distortion compensation, optical decision at the receiver in optical communication systems, spectral and temporal signal doubling, and frequency conversion.
\end{abstract}

\section{Introduction}

The use of photonic technologies for data processing in the all-optical domain has a strong potential for a variety of interesting applications in such diverse areas as optical telecommunications, metrology, optical sensing, microwave engineering, advanced microscopy image processing, optical computing, and many others. Advantages of processing the information in the all-optical domain include the large available bandwidth and the (potential) parallelism intrinsic to the optical approach, which translate into high-processing speeds. However, today electronic techniques of signal manipulation are advanced compared to all-optical processing devices, which are still at the research stage rather than under commercial development. A key feature of electronics, enabling many different applications, is the capability of generating electrical waveforms with arbitrary temporal profiles by use of simple integrated circuits. This capability is so far unmatched in the optical frequency range, where bulky and complex devices are required to shape the light fields. Hence, considerable knowledge still has to be accumulated and new methodologies need to be explored before a true breakthrough can be achieved in this field, allowing the range of functions and operations currently accomplished electronically, to be performed in the optical domain. Furthermore, by using optical rather than electronic processing additional functionality may be possible.

In order to realize such all-optical processing, nonlinear photonics is seen as a key technology. Optical fibre materials exhibit a nonlinear response to strong electric fields, such those of optical signals confined within the small fibre core. In high-speed optical communications such nonlinear effects generally degrade the integrity of the transmitted signal but the same effects can be used to realize a variety of optical functions that have practical applications in the field of lightwave technology. In this paper, we provide a snapshot of recent results and advances in the use of nonlinear effects in optical fibres for optical waveform generation and pulse shaping and in the applications of advanced pulse profiles in all-optical signal processing. We would like to note that the main attention in this paper will be focused on results obtained in our groups, and that it is not our intention here to comprehensively cover all the possible examples of fibrebased pulse shaping and signal processing. 


\section{Fibre Nonlinearities}

The third-order $\chi^{(3)}$ optical nonlinearity in silica-based single-mode (SM) fibres is one of the most important effects that can be used for all-optical signal processing. This happens not only because the third-order nonlinearity provides ultra-fast response times in the femtosecond range, but also because it is responsible for a wide range of phenomena such as third-harmonic generation, nonlinear refraction (Kerr nonlinearity), and stimulated Raman and Brillouin scattering [1]. The rapid recent developments of microstructured fibres with extremely small effective core areas and exhibiting enhanced nonlinear characteristics [2] and of fibres using materials with refractive indexes higher than that of the silica glass [3] have enabled dramatic reduction of the required fibre lengths for nonlinear processing compared to conventional fibres. Such progress has paved the way for the integration of fibre-based nonlinear processing functions on photonic chips.

The intensity dependence of the refractive index of a fibre gives rise to different effects depending on the shape of the input signal; the most widely studied effects are self-phase modulation (SPM) and cross-phase modulation (XPM). Considering the localized optical pulse evolution in a fibre medium and neglecting higher order effects in the fibre, pulse propagation can be described by the nonlinear Schrödinger (NLS) equation [4]:

$$
\psi_{z}=-i \frac{\beta_{2}}{2} \psi_{t t}+i \gamma|\psi|^{2} \psi,
$$

where $\psi(z, t)$ is the slowly varying pulse envelope in the comoving system of coordinates, $\beta_{2}$ and $\gamma=2 \pi n_{2} /\left(\lambda A_{\text {eff }}\right)$ are the respective group-velocity (second-order) dispersion (GVD) and Kerr nonlinearity coefficients of the fibre, $n_{2}$ is the nonlinear-index coefficient, $\lambda$ the central wavelength of the pulse, and $A_{\text {eff }}$ the effective core area. As a result of the nonlinear term in (1), upon propagation in the fibre the pulse acquires an intensity-dependent nonlinear phase shift as $\phi(z, t)=\gamma|\psi(0, t)|^{2} z$, namely, the frequency chirp $-\phi_{t}$. When the length scale associated with the pulse where the GVD effects take place: $L_{D}=T_{0}^{2} /\left|\beta_{2}\right|$ (with $T_{0}$ being some temporal characteristic value of the initial pulse) is much larger compared with both the fibre length and the nonlinear length $L_{\mathrm{NL}}=1 /\left(\gamma P_{0}\right)$ (with $P_{0}$ being the peak power of the initial pulse), the dispersive term in (1) can be neglected. In this case, the presence of a chirp causes a nonlinear broadening of the pulse spectrum. When two (or more) pulses copropagate inside a fibre, one still obtains an NLS equation for each pulse but these equations are coupled through XPM [4]. In this case, the nonlinear phase evolution for each pulse depends also on the power of the other pulses according to the expression $\phi_{i}(z, t)=$ $\gamma_{i}\left(\left|\psi_{i}(0, t)\right|^{2}+2 \sum_{j \neq i}\left|\psi_{j}(0, t)\right|^{2}\right) z$. XPM occurs only when the pulses overlap. Similar to the SPM case, XPM leads to additional nonlinear chirping and spectral shaping.

When the effects of chromatic dispersion are considered in combination with the Kerr nonlinearity, rich pulse dynamics arise from the interplay between dispersive and nonlinear effects depending on the sign of the dispersion and the relative magnitudes of the associated length scales. A well-known and fascinating example is the formation of optical solitons $[5,6]$ in the anomalous dispersion regime of a fibre $\left(\beta_{2}<0\right)$ as a result of a cooperation between GVD and SPM. On the contrary, in the regime of normal dispersion pulse dynamics are highly affected by the phenomenon of optical wave breaking [7-9].

In the next Section we will discuss some newly emerged qualitative features of the optical pulse evolution in a fibre under the combined action of dispersion and nonlinearity.

\section{Optical Pulse Generation and Shaping Using Fibre Nonlinearities}

Techniques for generating, controlling, manipulating, and measuring ultrashort optical pulses and specialized waveforms have become increasingly important in many scientific areas including, amongst others, ultrahigh-speed optical communications [10], optical signal processing, and biophotonics. To date, conventional picosecond and femtosecond pulse shaping in the optical domain have been implemented using devices, such as liquid crystal spatial light modulators $[11,12]$, wavelength-to-time mapping $[13,14]$, acoustooptic modulators, and electrooptical phase arrays, that impart user-specified spectral amplitude and phase to the pulse. Though powerful and flexible, this approach has the drawback of requiring a rather bulky and expensive apparatus, entailing a level of cost and complexity that is not often commensurate to the used laser system and target application. In order to fulfill telecommunication requirements, more compact techniques for pulse shaping have been developed, which include the use of super-structured fibre Bragg gratings [15], long-period fibre grating filters [16], and arrayed waveguide gratings that are well suited for line by line processing of high-repetition periodic pulse shapes $[17,18]$. However, in all these pulse shapers using linear techniques, the bandwidth of the input pulse determines the maximum bandwidth of the output. Indeed, a linear manipulation cannot increase the pulse bandwidth, and so to create shorter pulses nonlinear effects must be used. This limitation of the linear approaches becomes very stringent when synthesizing advanced pulse shapes such as rectangular pulses, for which a large optical bandwidth is required to reproduce accurately the spectral side lobes linked to the temporal compactness of the pulse. Moreover, in those applications that require spectral narrowing, linear filtering introduces a power penalty that is at least proportional to the ratio of the target spectrum to the input one.

The combination of third-order nonlinear processes and chromatic dispersion in optical fibres can provide efficient new solutions to overcome the aforementioned limitations. The pulse-shaping examples discussed below highlight some of the advantages offered by a passive, all-optical nonlinear fibre-based approach to pulse shaping.

3.1. Ultrahigh Repetition Rate Pulse Train Generation. As a first illustration of the possible nonlinear pulse shaping mechanisms in optical fibres, we review here the generation of ultrashort pulse trains with very high repetition rates 
resulting from the passive evolution of optical pulses in an anomalously dispersive fibre in the presence of Kerr nonlinearity. As mentioned in the previous Section, in the anomalous GVD regime of a fibre, the NLS equation (1) admits families of soliton solutions. While fundamental solitons are characterized by an exact balance between the GVD and SPM effects $\left(N=\sqrt{L_{\mathrm{D}} / L_{\mathrm{NL}}}=1\right)$, in the case of higher order solitons $(N>1)$ SPM dominates initially but GVD soon catches up and leads to pulse contraction. This stage of temporal compression can be used for generating pulses with ultrashort durations [19-21]. However, the compressed pulses exhibit a low-amplitude structure outside the central lobe. An alternative technique that prevents sidelobe formation relies on the progressive evolution of the peak power and temporal duration of a fundamental soliton in a distributed amplifier or a dispersion decreasing (DD) fibre [22, 23]. Step-like or comb-like [24] dispersion profiled structures based on carefully chosen sets of fibres have been successfully used to emulate continuous DD fibre profiles.

The generation of high repetition rate pulse sources has largely benefited from the nonlinear compression effect taking place in the anomalous dispersion region, and several works have demonstrated the nonlinear reshaping of a sinusoidal beat-signal into well-separated pulses, the pulse repetition rate being simply determined by the frequency separation between the two continuous-wave (CW) laser sources. This technique has been successfully demonstrated with various experimental setups including DD fibres, adiabatic Raman compression in standard fibres, and steplike and comb-like dispersion profiled fibres. More recently, this nonlinear compression effect has been observed through a multiple four-wave mixing (FWM) process in a single anomalously dispersive fibre, and repetition rates ranging from a few tens gigahertz to 1 terahertz have been achieved [25]. In the frequency domain, such nonlinear compression can be viewed as the result of a modulation instability [26] experienced by the initial beat signal during propagation in the fibre, with the generation of new sidebands via FWM: two pump photons at $\omega_{1}$ and $\omega_{2}$ interact to generate two firstorder sideband photons at $\omega_{1}-\Omega$ and $\omega_{2}+\Omega, \Omega=\omega_{2}-\omega_{1}$ being the pump frequency detuning. High-order sidebands are then generated due to multiple FWM interactions. We note that a powerful alternative technique for realizing high-repetition-rate short-pulse sources relies on harmonic passive mode locking of fibre lasers [27-30]. The advantage of laser cavities as such compared to nonlinear pulse shaping in one-stage fibre systems is the possibility of achieving lower pulse train duty cycles. However, laser cavities have the drawbacks that they require mode locking, they cannot be referenced to an external clock directly and are not very suitable for the generation of pulses with ultrahigh repetition rates above a hundred gigahertz.

A typical experimental setup for the generation of high repetition rate pulse trains at telecommunication wavelengths is sketched in Figure 1(a). The initial beat signal is easily obtained via temporal superposition of two CWs with slightly different wavelengths delivered by external cavity lasers or a single CW directly modulated by an intensity modulator. A phase modulator driven at a frequency of a

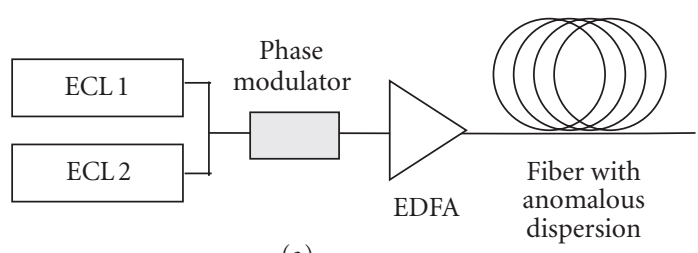

(a)
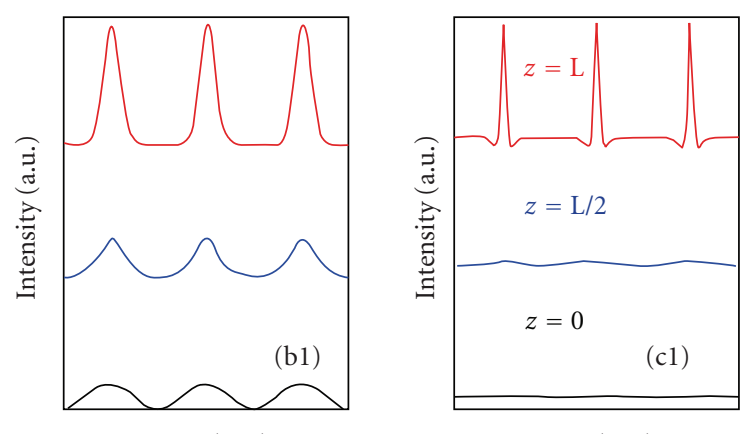

Time (a.u.)

Time (a.u.)

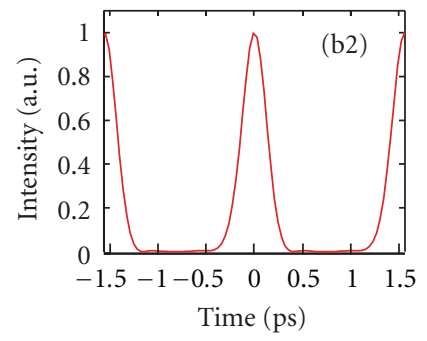

(b)

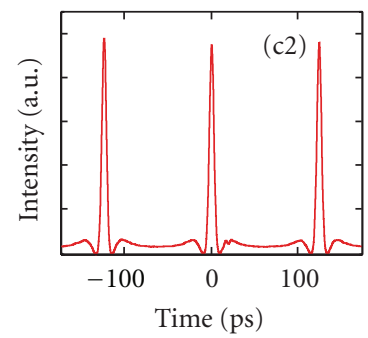

(c)
FIGURE 1: (a) Experimental setup for high repetition rate pulse train generation. (b, c) Results obtained for the optimum output shape and for the best compression, respectively. The temporal intensity profiles at different stages as predicted by numerical simulations (subplots 1) are compared to the experimental results (subplots 2) obtained by the FROG technique (b2-results adapted from [25]) or by an optical sampling oscilloscope (c2-results adapted from [31]).

few hundreds of megahertz is used to avoid the deleterious effects of Brillouin back scattering. The beat signal is then amplified up to the Watt level by an erbium-doped fibre amplifier (EDFA) and propagated through a suitable optical fibre with anomalous dispersion to realize the nonlinear reshaping. According to the pulse source parameters that are chosen (pulse repetition rate, initial peak power, nonlinearity and dispersion coefficients of the compression fibre, and fibre length), various output pulse shapes can be obtained. Intensity and phase measurements based on the frequency resolved optical gating (FROG) technique [32] show that high-quality compressed pulses with a Gaussian intensity profile and nearly constant phase can be achieved at a typical duty cycle of $1 / 5[25,33]$ (Figure 1(b)). Lower duty cycles can still be obtained with a single fibre, but at the expenses of increased sidelobes in the wings of the pulses [34]. Such sidelobes can be partly avoided by using specially designed 
arrangements of segments of fibres [35-37] or other comblike structures.

We would like to note that the reshaping of a sinusoidal beat signal into ultrashort structures can be understood in terms of the nonlinear dynamics of "Akhmediev breathers" $[38,39]$. In this context, the extreme spatiotemporal localization that can be observed (see, e.g., Figure 1(c)) is explained as a result of the formation of Peregrine solitons [31, 40], mathematical solutions of the standard NLS equation that have been first derived in 1983 in the hydrodynamics context [41] but have never been demonstrated experimentally before 2010, and using experiments in optical fibres. These observations offer a promising way to better understand the problem of the occurrence of rogue waves in optics and hydrodynamics $[42,43]$.

\subsection{Generation of Parabolic Pulses in Active and Passive} Fibres. Strong pulse reshaping can also occur in the normal regime of dispersion of a fibre. An important example that has generated a great deal of attention in the last decade is the occurrence of self-similar dynamical effects in the nonlinear propagation of ultrashort pulses in normally dispersive (ND) optical fibre amplifying media [44]. Results have demonstrated a fundamentally new operating regime where the interaction of Kerr nonlinearity, dispersion, and optical gain is exploited to generate a particular class of pulses with a parabolic intensity profile and a strictly linear frequency chirp. These pulses then propagate in a self-similar manner, holding certain relations (scaling) between pulse power, duration, and chirp parameter. The results for the self-similar parabolic pulses are obtained by assuming that a fibre amplifier can be described by the NLS equation with gain

$$
\psi_{z}=-i \frac{\beta_{2}}{2} \psi_{t t}+i \gamma|\psi|^{2} \psi+\frac{g}{2} \psi
$$

where $g$ is the distributed gain coefficient. Using a selfsimilar ansatz and standard amplitude/phase decomposition, one can find self-similar parabolic pulse solutions in the quasiclassical limit of $(2)\left(\beta_{2}\left|(|\psi|)_{t t}\right| /\left(2 \gamma|\psi|^{3}\right) \ll 1\right)$, whose dynamic evolution is summarized as $[45,46]$

$$
\begin{gathered}
|\psi(z, t)|=a(z) \sqrt{1-t^{2} / \tau(z)^{2}} \theta(\tau(z)-|t|), \\
\arg \psi(z, t)=b(z) t^{2}+\phi_{0}(z), \quad a(z)=a_{0} e^{g z / 3}, \\
\tau(z)=\tau_{0} e^{g z / 3}, \quad b(z)=-\frac{g}{6 \beta_{2}},
\end{gathered}
$$

in the case of constant gain. Here, $\theta(x)$ is the Heaviside function. The amplitude term indeed reveals a parabolic intensity profile, and the phase term a parabolic phase modulation or linear chirp with chirp rate $b / 2$. The peak amplitude and width of the pulse scale from their initial values, and the initial values depend only on the initial energy and amplifier parameters. These solutions possess the remarkable property of being a global attractor to the system for arbitrary initial conditions [47, 48]. By analogy with the well-known stable dynamics of solitary waves or solitons, these self-similar parabolic pulses have come to be known as "similaritons". In contrast to solitons, similaritons can tolerate strong nonlinearity without wave breaking. The normal GVD effectively linearizes the accumulated phase of the pulse allowing for the spectral bandwidth to increase without destabilizing the pulse [49]. The unique properties of similariton pulses have motivated many theoretical and experimental studies (see, e.g., [46, 48, 50-53]).

Experimental demonstrations of similariton generation relying on amplification from either rare-earth doping (with ytterbium [45, 56-59] or erbium [51] dopants) or Raman scattering $[50,54,60]$ have been achieved, confirming the potential of this method especially when dealing with the generation of high-power ultrashort pulses. Thanks to the high level of linearity of the chirp developed during parabolic amplification and as long as the impact of third-order fibre dispersion is moderate $[52,61,62]$, it is possible to efficiently compensate for the chirp slope, so that compressed pulses with very low substructures and temporal durations much shorter than the initial seed pulse can be obtained $[45,51$, 56-59]. As an illustration, Figures 2(a) and 2(b) show the setup and results of an experiment where similaritons are generated through Raman amplification at telecommunication wavelengths [54]. Moreover, recent fibre lasers that use self-similar pulse shaping in the normal dispersion regime have been demonstrated to achieve high-energy pulses [6366].

In addition to fibre amplifiers and lasers, stable similariton pulses can be generated in passive fibres provided a suitable longitudinal variation of the dispersion is introduced [55, 67-69] (Figure 2(c)). This approach is based on the observation that a longitudinal decrease of the normal dispersion is formally equivalent to linear gain. Recently, a simple approach to the generation of parabolic pulses that uses progressive nonlinear pulse reshaping in a ND fibre with fixed dispersion has been demonstrated [70]. However, in contrast with the asymptotic similariton solutions obtained in fibre amplifiers, the generated parabolic waveforms represent transient states of the nonlinear pulse evolution in the passive fibre medium [71]. As such, they have a finite life distance that depends sensibly on the initial conditions (pulse shape, energy, and chirp profile). Nevertheless, stabilization of the parabolic features is possible by use of a second propagation stage in a fibre with specially adjusted nonlinear and dispersive characteristics relative to the first fibre [70, 71].

3.3. Generation of Triangular Pulses. Parabolic shapes are not the only pulse waveforms that can be generated in a passive ND fibre. Recently, the combination of pulse prechirping and nonlinear propagation in a section of ND fibre has been introduced as a method for passive nonlinear pulse shaping, which provides a simple way of generating various advanced field distributions, including flat-top- and triangular-profiled pulses with a linear chirp [71]. In this scheme, Kerr nonlinearity and GVD lead to various reshaping processes of an initial conventional pulse (e.g., a Gaussian pulse) according to the chirping value and power level at the entrance of the fibre. In particular, 


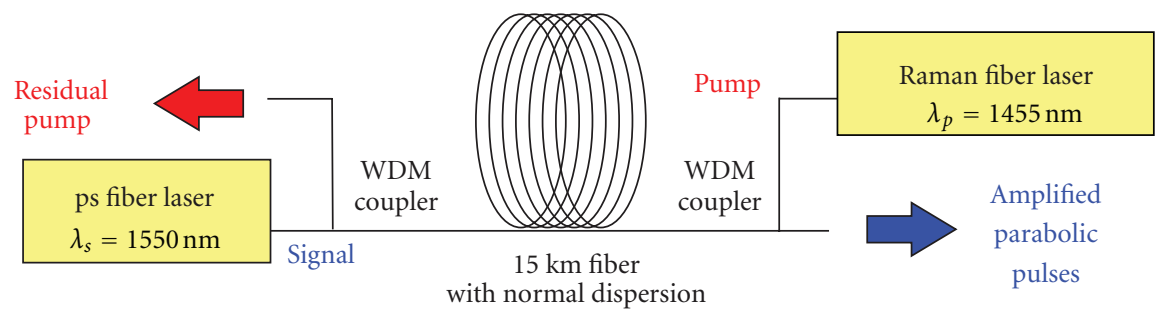

(a)
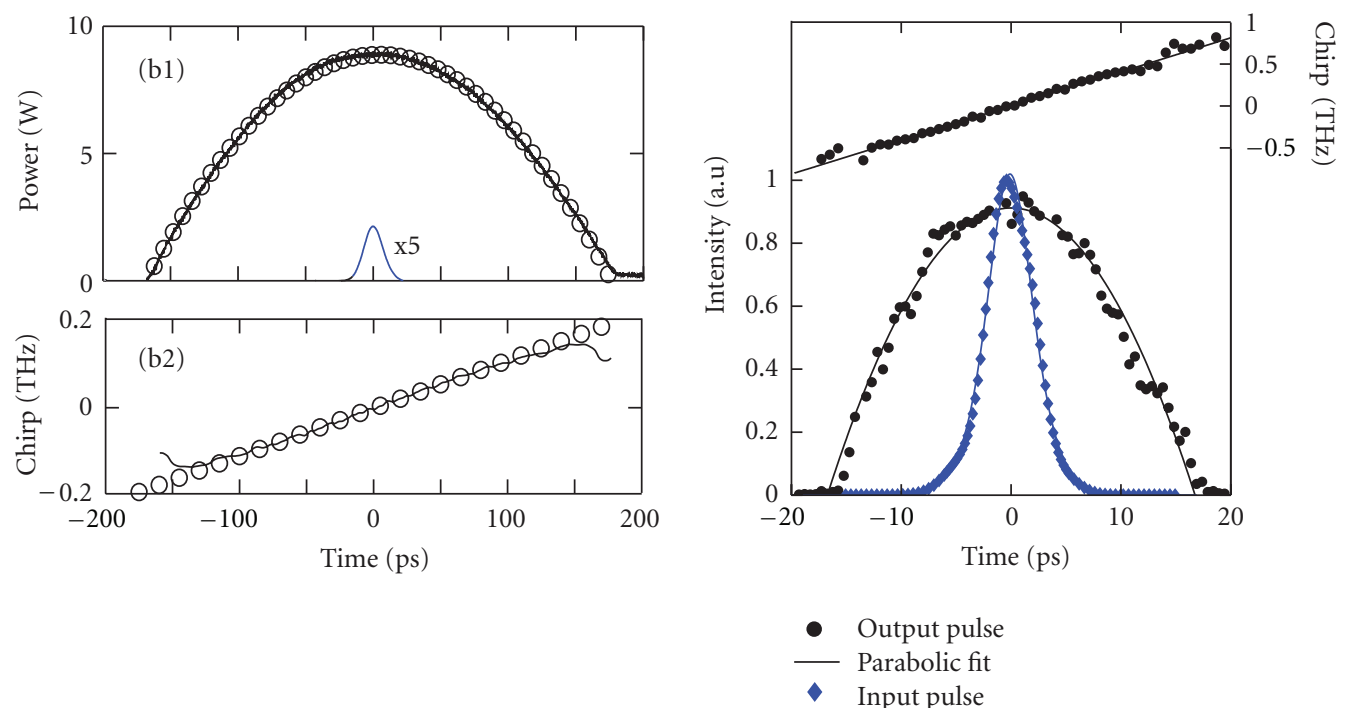

(b)

(c)

Figure 2: (a) Experimental setup for parabolic pulse generation through Raman amplification at telecommunication wavelengths. (b) Results obtained in the Raman amplifier (adapted from [54]): temporal intensity profile (subplot b1) and chirp profile (subplot b2) of the output pulse from the amplifier. The experimental results (solid black lines) are compared to a parabolic and a linear fit (open black circles), respectively. The intensity profile of the initial pulse is also shown (solid blue line). (c) Results obtained in a ND decreasing fibre (adapted from [55]): experimental temporal intensity and chirp profiles at the fibre output (black circles) compared to a parabolic and a linear fit (solid black lines), respectively. The initial intensity profile is also shown (blue diamonds).

triangular pulses can be generated for a positive initial chirp parameter (using the definition $i b t^{2}$ for the phase profile) and sufficiently high energies.

These theoretical results have been confirmed experimentally by intensity and phase measurements of the generated pulses [72] (Figure 3(b)). In the experimental setup used (Figure 3(a)), the control of the pulse prechirping value was realized by propagation through different lengths of standard SM fibre with anomalous GVD, which imposed a positive chirp parameter on the pulse. The prechirped pulses were amplified to different power levels using an EDFA and then propagated through a ND fibre to realize the pulse reshaping.

Different nonlinear dynamics may lead to the generation of triangular pulses in a passive ND fibre. Indeed, it has been recently shown qualitatively and numerically that temporal triangular intensity profiles can result from the progressive reshaping of initially parabolic pulses driven by the fourthorder dispersion (FOD) of the fibre [53]. The overall temporal effect of FOD on parabolic pulse propagation is to stretch and enhance the power reduction in the pulse wings, leading to a triangular profile. Furthermore, the possibility of triangular pulse shaping in mode-locked ring-cavity fibre lasers has been first reported in [73]. It has been numerically demonstrated that for normal net dispersion, formation of two distinct steady-state solutions of stable single pulses can be obtained in a laser cavity in different regions of the system parameter space: the previously known similariton [63] and a triangular-profiled pulse with a linear frequency chirp.

\subsection{Coherent Continuums for Optical Telecommunications.} One of the most well-known examples of pulse reshaping in the spectral domain is in supercontinuum (SC) generation. SC generation in optical fibres, and more specifically, in photonic crystal fibres with enhanced nonlinearity, is an established technique for producing broadband light sources in a wide range of research fields including metrology, biophotonics, and optical telecommunications [74, 75]. In telecommunications, the broadened spectrum can be spectrally sliced to generate wavelength multiplexed pulse trains [76-78]. The principle of operation of a multiwavelength source is illustrated in Figure 4(a). An initial high-power 


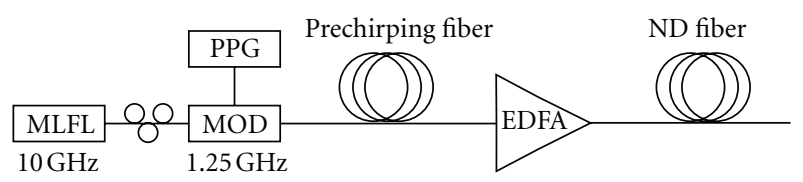

(a)
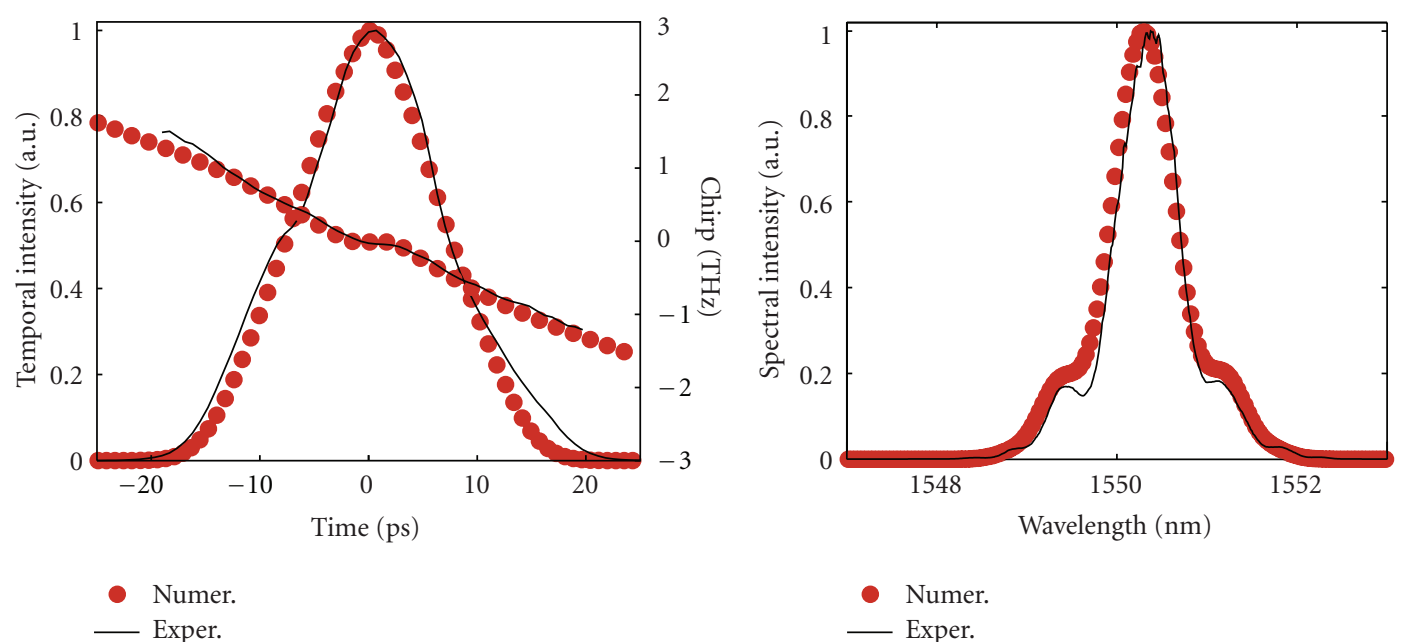

(b)

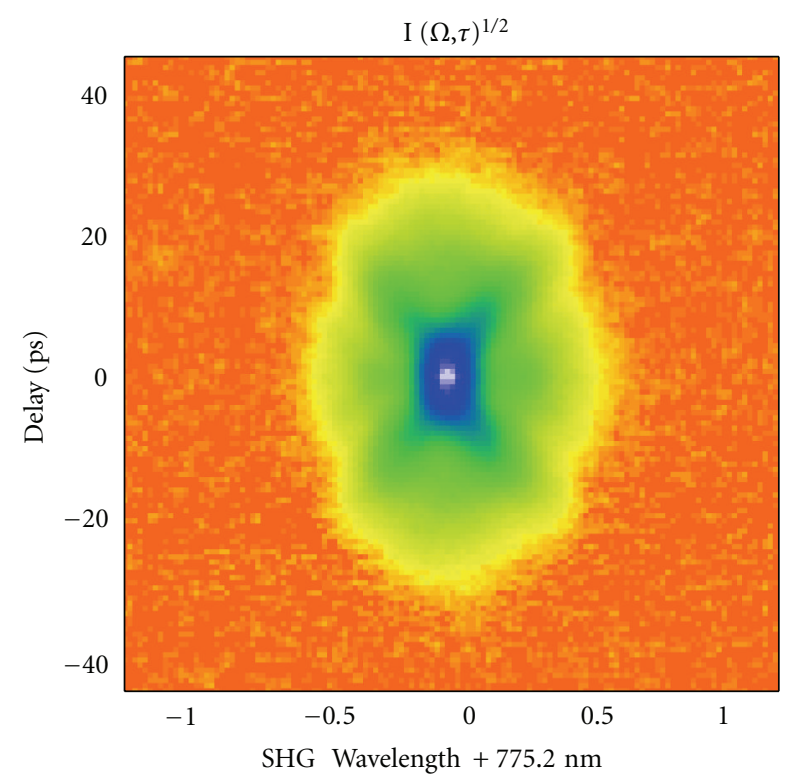

(c)

FIgURE 3: (a) Experimental setup for triangular pulse generation. (b) Experimental and numerical temporal intensity and chirp ( $\left.\phi_{t}\right)$ profiles retrieved from second-harmonic generation (SHG) FROG (left) and optical spectra (right) of the generated pulses for an example length of prechirping fibre. (c) SHG-FROG spectrogram of the pulse in (b). Results adapted from [72].

mode-locked picosecond or femtosecond pulse train with a repetition rate of several gigahertz is spectrally broadened through SPM in a kilometer-long highly nonlinear (HNL) fibre. At the fibre output, the spectrum is sliced by adequate bandpass filters (BPFs) to obtain pulse trains that have the same repetition rate as the initial train but new central frequencies.
Typically the broadest spectra are generated in anomalously dispersive fibres, where the broadening mechanism is strongly influenced by soliton dynamics [80]. However, solitonic effects and modulation instability lead to decreased temporal coherence and spectral flatness of the continuum generated in the anomalous dispersion regime [81]. By contrast, the nonlinear pulse evolution in ND fibres leads 


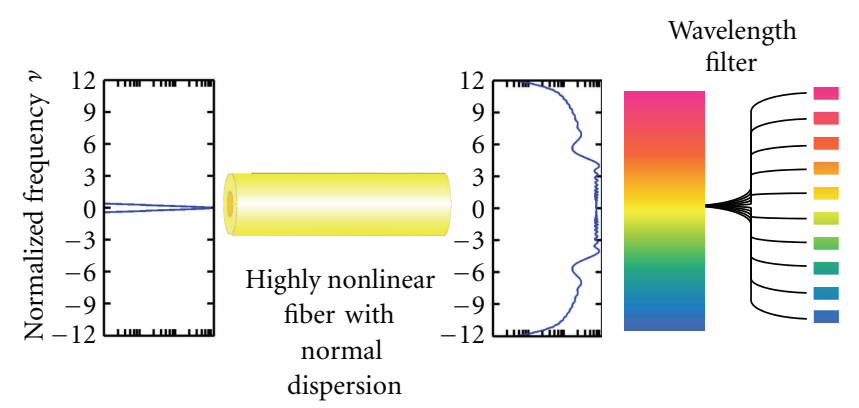

(a)

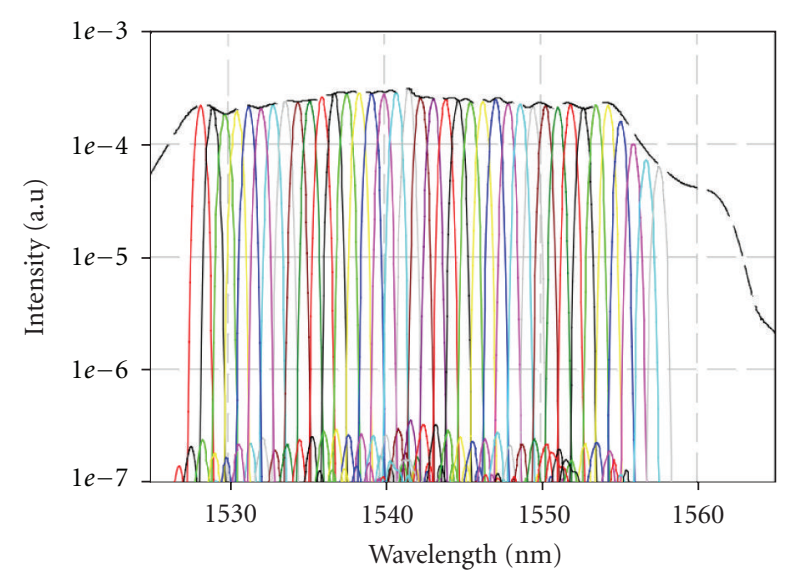

(b)

FIGURE 4: (a) Principle of operation of a multiwavelength picosecond source. (b) Experimental spectrum of a parabolic-shaped pulse after propagation in a ND-HNL fibre (black dashed line) and superposition of the measured spectra of 38 sliced channels. Results adapted from [79].

to flatter spectra and improved temporal coherence, but at the expenses of relatively narrower spectra [82, 83]. Hence frequency broadening using a ND fibre is an attractive choice whenever a moderate amount of broadening alongside good spectral and temporal stability of the output pulses are targeted. However, the main limits to spectral pulse quality in the normal dispersion regime are the spectral ripple arising from SPM of conventional laser pulses, and the effects of optical wave breaking which may lead to significant change in the temporal pulse shape and severe energy transfer into the wings of the spectrum. These effects can in principle be avoided by using preshaped input pulses with a parabolic temporal intensity profile, which would preserve their shape whilst propagating within the fibre and, thus, result in spectrally flat, highly coherent pulses. Continuum with lowspectral ripple and high-energy density in the central part is indeed achievable through parabolic pulse propagation in ND-HNL fibre [79], and low-noise multiwavelength picosecond sources covering the whole C-band of optical telecommunications have been demonstrated based on this technique $[12,16,79]$ (Figure 4(b)).

\section{Applications of Parabolic and Triangular Pulses in Signal Processing}

Applications of parabolic pulses have not been limited to ultrashort high-power pulse generation and highly coherent continuum sources, but several optical processing techniques have taken advantage of their specific features. These include optical regeneration $[84,85]$ including pulse retiming [86], prereceiver nonlinear processing in the optical domain $[87$, 88 ], and mitigation of linear waveform distortions [89, 90]. The simple intensity profile of triangular pulses is also highly desired for a range of processing applications, including time domain add-drop multiplexing [91, 92], wavelength conversion [93-95], optical signal doubling [96], time-to-frequency mapping of multiplexed signals [97], and enhanced spectral compression $[98,99]$. In this Section, we will review some of these applications.

4.1. $2 R$ Regeneration. As a first example, here we discuss the usage of parabolic pulses in $2 \mathrm{R}$ (reamplification, retiming) signal regeneration in an extension of the technique proposed by Mamyshev [100]. The method relies on SPM in a nonlinear medium (fibre) and subsequent offset spectral filtering to suppress the noise in the zero-bit slots and the amplitude fluctuations in the one-bit pulses of return-tozero (RZ) optical data streams. In the schematic of the Mamyshev regenerator depicted in Figure 5(a), the signal to be regenerated is first amplified to the optimum power level, and an optical BPF is also used to reduce the amplified spontaneous emission noise power. Then spectral broadening is achieved in a HNL fibre, and the broadened spectrum is partially sliced by a second BPF, which is shifted with respect to the input signal frequency in order to transmit SPMgenerated frequencies and reject the original spectrum. The central frequency and output pulse shape of the regenerated signal are determined by the central frequency and spectral profile of the offset filter, respectively. The SPM phase shift experienced in the HNL fibre depends on the instantaneous power (cf. Section 2). Hence, a high-intensity pulse induces more SPM phase shift and spectrally broadens more than the low-intensity noise [100]. Filtering the new SPM-generated frequencies with the offset filter leads to a power transfer function for the pulses and the noise which is close to a binary one [101].

Spectral filtering of similariton pulses can improve the ability of the Mamyshev regenerator to eliminate spurious noise pulses as well as to simultaneously reduce any fluctuations in the signal one-bit level [84]. This ability relies on the specific features of self-similar amplification. Indeed, a spectrum broadened through self-similar amplification has a smooth profile (cf. Section 3.4), whereas a SPM-broadened spectrum is generally accompanied by oscillatory structures 


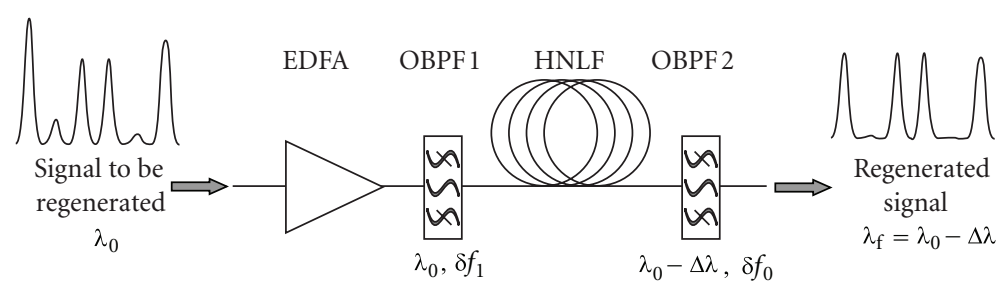

(a)

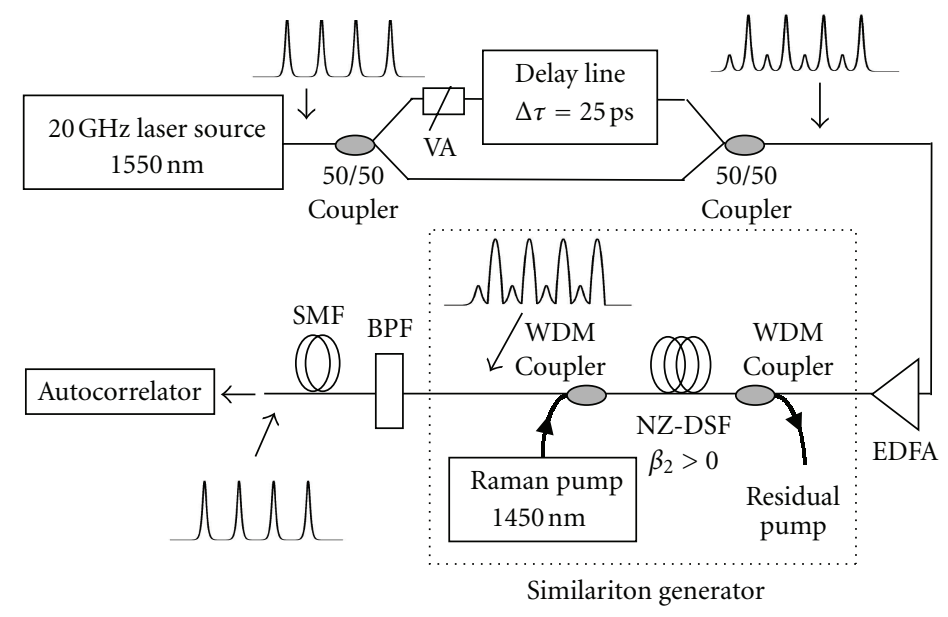

(b)
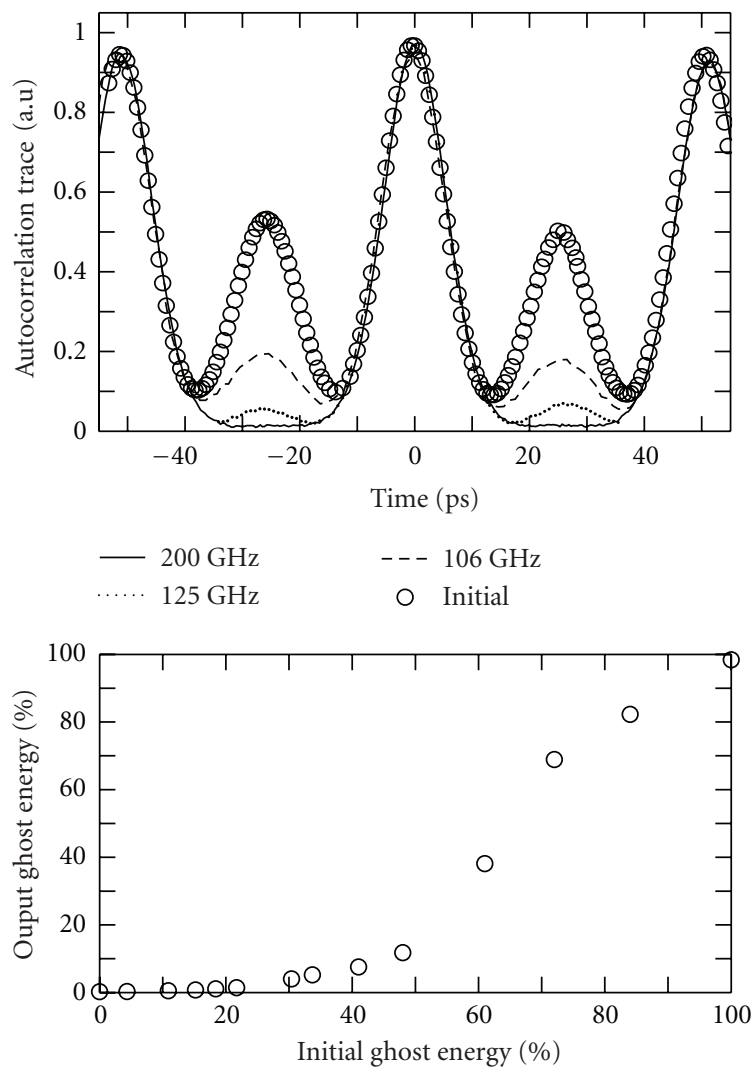

(c)

FIGURE 5: (a) Schematic of an optical regenerator based on SPM and filtering (after [100]). (b) Experimental setup for 40 Gbit/s similaritonbased regeneration. (c) Top, autocorrelation traces of the pulse train at the Raman amplifier input (circles) and output for different values of the filter offset (dashed, dotted, and solid curves). Bottom, output versus input proportion of the spurious noise pulse energy with respect to the ones energy for a fixed filter offset. Results adapted from [84].

covering the entire frequency range. Moreover, the spectrum and chirp obtained by SPM both depend on the details of the initial pulse (shape, chirp, and energy), leading to some amplitude and time fluctuations of the regenerated pulses. In contrast, the similariton-based spectral broadening and similariton-induced chirp depend only on the initial pulse energy and/or amplifier parameters, respectively (cf. Section 3.2), so that both amplitude and timing jitter are reduced. In particular, the energy-dependent spectral broadening is responsible for efficient discrimination of highenergy pulses (ones) from low-energy pulses (zeros) with the offset BPF. An implementation of the similariton-based regeneration technique at $40 \mathrm{Gbit} / \mathrm{s}$ data rate where similariton pulses are generated through distributed Raman amplification in a ND fibre is illustrated in Figures 5(b) and 5(c). It is worth noting here that a distributed amplification is helpful in increasing the energy yield of the regenerator which is a highly dissipative element [102].

4.2. Linear-Distortion Compensation. As a second example of the application of parabolic pulses in signal processing, we review here the realization of a time-domain alloptical Fourier transformation (OFT) technique. Timedomain OFT in which the spectral profile is converted into a waveform in the time domain makes it possible to eliminate the waveform distortion caused by linear perturbations in optical fibres, such as higher order dispersion, jitter and polarization-mode dispersion [103-106]. Timedomain OFT is realized ideally by applying a quadratic temporal phase modulation (time lens) to a distorted pulse: 
$\psi_{\text {chirp }}(t)=\psi(t) \exp \left(i b t^{2}\right)$ and passing the linearly chirped pulse through a dispersive medium such as a short piece of fibre. When the accumulated dispersion of the medium $D=\beta_{2} L$ equates the inverse of the chirp coefficient $b$ (i.e., when the chirp is fully compensated), the output waveform is proportional to the spectral amplitude before OFT: $\chi(t)=\sqrt{i /(2 \pi D)} \exp \left(-i b t^{2}\right) \widetilde{\psi}(t / D)$, where $\widetilde{\psi}(\omega)$ is the Fourier transform of $\psi(t)$, and the frequency $\omega$ is converted into time $t$ through the relationship $\omega=t / D$. Since generally the spectral envelope profile has no distortion even if its time-domain waveform is distorted by linear perturbations, this frequency-to-time mapping indicates that a distortionfree pulse waveform can be obtained in the time domain after OFT. In [103-106], this compensation technique was investigated by using electrooptic phase modulators. But this way the quadratic modulation region was restricted to around the center of the sinusoidal modulation imparted by the phase modulator. Thus, any parts of the pulse extending beyond the quadratic region could not be properly transformed, and only the section of the target pulse sitting within the quadratic region could be compensated (Figure 6).

The use of XPM with parabolic pulses as a time lens has been recently proposed as a promising way to overcome the aforementioned limitation $[89,90]$. Indeed, the chirp induced by XPM on the target pulse is proportional to the gradient of the intensity profile of the parabolic control pulse: $-\phi_{t}=-2 b t, b=\mp 2 \gamma a^{2} L / \tau^{2}$, so it is exactly linear and thus ideal OFT can be achieved by complete chirp compensation. Here, $a$ and $\tau$ are the respective peak amplitude and characteristic width of the parabolic pulse (cf. Section 3.2), and the sign $\mp$ is for either a standard "bright" or a dark parabolic pulse [17]. The parabolic XPM-based OFT technique has been successfully experimentally demonstrated to achieve cancellation of the third-order dispersion-induced distortion of short-optical pulses $[89,90]$.

4.3. Optical Decision at the Receiver. In this section, we review a possible application of parabolic/flat-top pulses to the improvement of the signal bit-error rate (BER) in an optical communication system by using optical decision before the receiver. An attractive approach to the design of the optical receiver for high-speed fibre communication systems using RZ data formats is based on employing an additional all-optical decision element (ODE) just before the conventional receiver $[87,107-109]$. In this way, the first decision is carried out in the optical domain, thus improving the quality of the received signal. The attractive possibility of improving the BER by using optical devices has been studied in some recent works $[88,110,111]$. The conceptual basis of the BER improvement by optical devices comes from the information theory: an intrinsic BER improvement is possible whenever the decision performed by the device in the optical domain is based on information that is different from the information used by the decision circuit of the (suboptimal) receiver in the electrical domain. In [88] an application of this general concept was demonstrated, based on using an advanced optical receiver enhanced by a nonlinear ODE. The example of an ODE scheme used in [88] exploits the nonlinear mechanism that leads to the formation of parabolic pulses and the resultant flattening of the signal waveform (cf. Section 3.2). The method is based on the nonlinear reshaping of a pulse towards a broad and flat temporal waveform that can occur upon propagation in a ND fibre [9] and subsequent slicing of the pulse temporal profile by an optical temporal gate (e.g., an amplitude modulator with a sharp nonlinear transfer function or a nonlinear optical loop mirror provided with a clock) [85] or XPM-based techniques.

The basic mechanism that is responsible for BER improvement in this scheme can be understood in the following way. The pulse temporal broadening and flattening in the ND fibre permits to recover the center of the bit slot for those pulses that are significantly shifted from the bit slot center in the input pulse train because of timing jitter (Figure 7(a)). Such strongly time-shifted pulses, once they are converted into electrical pulses and arrive at the decision point, might be missed by the threshold detector in those conventional receivers that use a relatively small fraction of the bit period. In contrast, the bit slot recovery in the ND fibre enables such pulses to be recognized as marks in the ODE-enhanced receiver. The slicing of the central portion of the broadened pulse temporal profile by the temporal gate enables efficient suppression of the timing jitter of the pulses which, in turn, improves the receiver sensitivity [87]. This approach is illustrated in Figure 7(b), which shows that BER improvement over the conventional scheme, indeed, can be achieved using nonlinear signal processing in the optical domain before the conventional receiver. It is worth noting that the same processing technique based on temporal slicing of nonlinearly flattened pulse waveforms can also be used in all-optical 3R (2R + re-timing) signal regeneration [85].

4.4. Optical Signal Doubling and Frequency Conversion. Here we discuss the use of triangular pulses in the context of optical signal doubling and frequency conversion. A technique of copying optical pulses in both the frequency and time domains based on a combination of either XPM with a triangular pump pulse or SPM of a triangular pulse in a nonlinear Kerr medium and subsequent propagation in a dispersive medium was introduced in [96] and experimentally demonstrated in [95]. In this scheme, spectral doubling of an optical signal is made possible by the XPM or SPM phase shift generated by a temporal intensity profile with a linear gradient: $\phi(t)=\phi_{0}(1-|t / \tau|), \phi_{0} \propto a^{2} \gamma L$ ( $a$ and $\tau$ being the peak amplitude and characteristic width of a triangular pulse, resp.), which translates into a constant and distinct (opposite sign) frequency chirp induced onto the leading (downshifted) and trailing (upshifted) edges of the signal pulse. As a result of this constant and distinct chirp, under certain conditions the pulse spectrum develops a structure consisting of two equal peaks. The separation between the two spectral peaks increases with the maximum nonlinear phase shift $\phi_{0}$, while their form is almost preserved. Under subsequent propagation of the modulated signal pulse in a linear dispersive medium, the produced spectral separation of the pulse components leads to their subsequent separation in time, thus allowing temporal replication of the pulse. In the case of normal dispersion, the pulse develops into a 


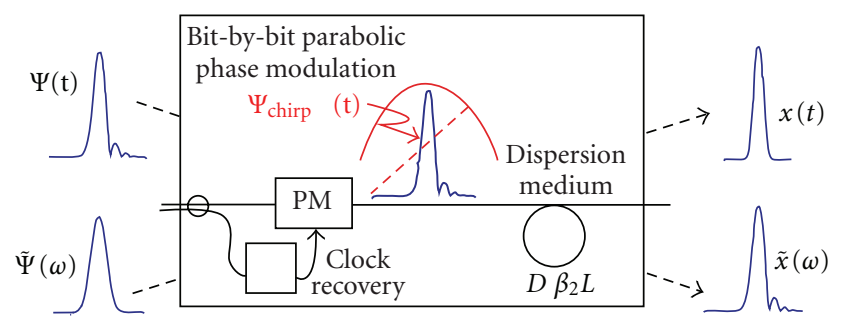

FIGURE 6: Schematic diagram of time-domain OFT (after [90]).

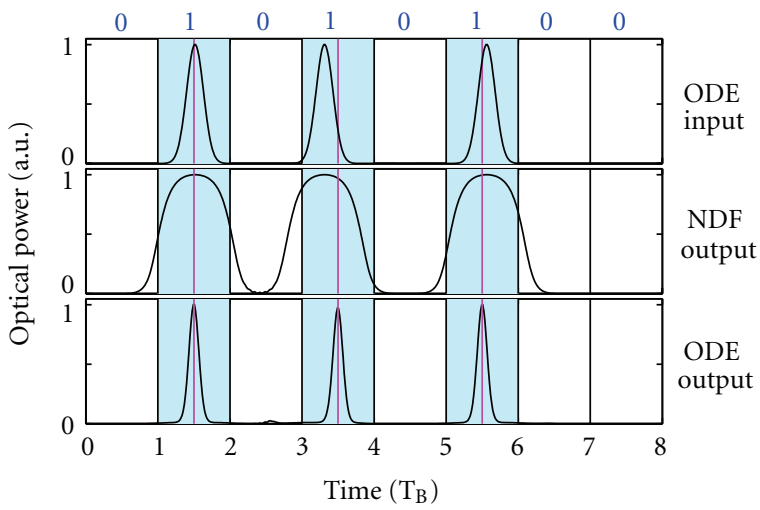

(a)

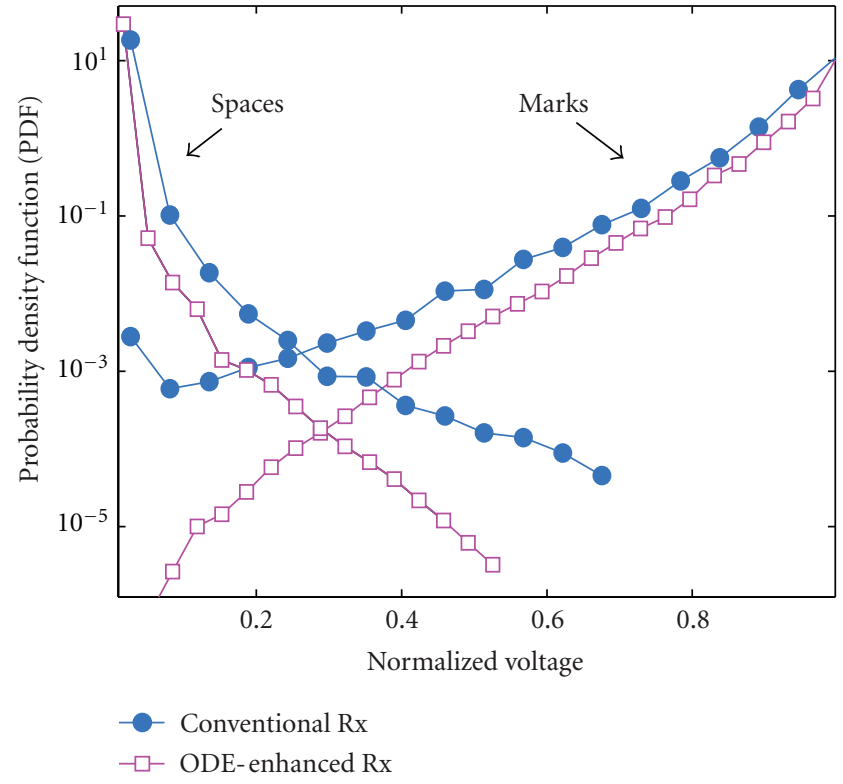

(b)

Figure 7: (a) Schematic illustration of the optical decision at an ODE-enhanced receiver. (b) Numerically calculated probability density functions for the voltage of the marks and spaces in the conventional and ODE-enhanced receivers for a $40 \mathrm{Gbit} / \mathrm{s}$ RZ input signal with a random jitter of the pulse temporal position. Results adapted from [88].

structure consisting of two identical pulses where the original pulse splits down the middle. The two pulse parts separate in time while experiencing dispersive broadening. The relative velocity of separation is proportional to the separation between the two peaks of the nonlinearly modulated pulse spectrum. Pulse splitting and separation occur also in the case of anomalous dispersion after an initial stage of pulse compression. Exact analytic expressions for the nonlinearly modulated pulse spectrum and the pulse temporal intensity distribution clearly show the features described above [96]. Applying spectral filtering to the XPM/SPM modulated signal is also possible to realize efficient and clean frequency conversion [93-95].

Figure 8 shows results of the experiment reported in [95]. Similarly to [72], triangular pulses were generated by passive nonlinear reshaping in a ND fibre. Spectral pulse doubling was realized through the SPM experienced by the triangular pulses over their life distance in a HNL fibre. The output SPM-shaped spectrum from the HNL fibre was sent into either a linear standard SM fibre segment to realize temporal pulse doubling or a wavelength demultiplexer filter for frequency conversion processing.

\section{Conclusion}

We have provided an overview of several recent examples of the use of nonlinear phenomena in optical fibres for the generation and shaping of optical pulses. We have discussed the generation of ultrashort pulse trains with very highrepetition rates in the anomalous dispersion regime of a fibre, the generation of parabolic-shaped pulses in active and passive ND fibres, the generation of pulses with triangular intensity profiles in passive ND fibres, and the generation of coherent continuums for optical telecommunications in ND fibres. Examples of new techniques or improvements in existing techniques of all-optical nonlinear fibre-based signal processing enabled by specialized pulse waveforms have been reviewed. Specifically, we have presented applications of parabolic pulses in optical signal regeneration, compensation of linear waveform distortions, and signal postprocessing in 

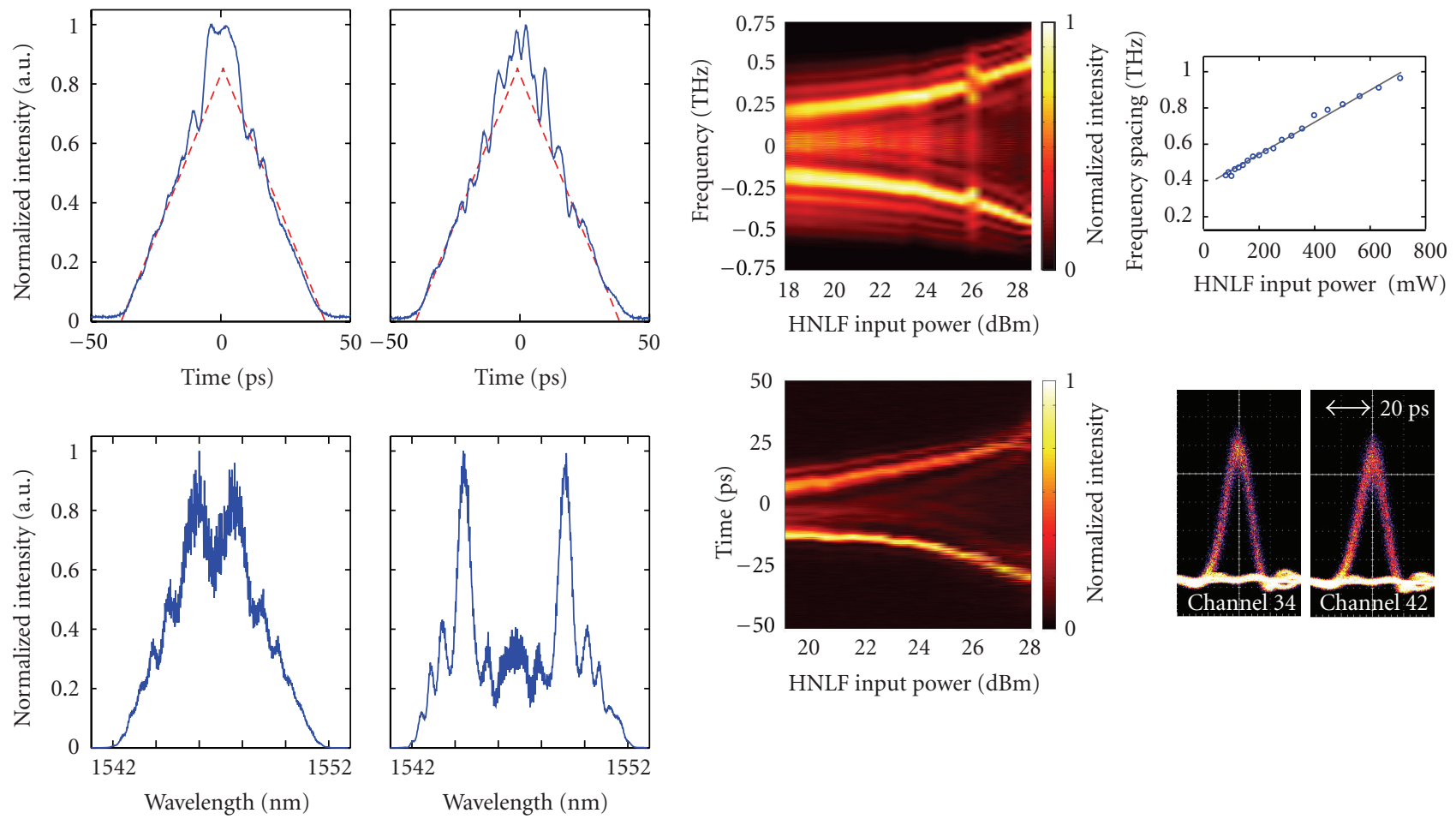

(a)

(b)

FIgURE 8: (a) Temporal (top) and spectral (bottom) intensity profiles after the ND reshaping fibre (left) and after the HNL fibre (right). (b) Top, evolution of the output spectrum from the HNL fibre with the input power (left) and dependence of the frequency spacing between the two spectral peaks on the input power (right). Bottom, evolution of the temporal intensity profile at the output of the linear SM fibre with the input power to the HNL fibre (left) and eye diagrams of a $10 \mathrm{Gbit} / \mathrm{s}$ pulse train obtained after spectral filtering for the ITU channels 34 and 42. Results adapted from [95].

optical communication systems. Applications of triangular pulses in optical signal doubling and frequency conversion have also been discussed.

\section{Acknowledgments}

The authors would like to acknowledge and thank our colleagues, namely, Prof. Sergei K. Turitsyn, Dr. Paul Harper, Dr. Anton I. Latkin, Prof. Keith J. Blow, Dr. Julien Fatome, Dr. Kamal Hammani, Dr. Bertrand Kibler, Prof. Guy Millot, and Prof. John M. Dudley and the Optoelectronics Research Center (Southampton, UK). The authors also acknowledge support from the British Council (Alliance Grant 10.002), the Ministère des Affaires Étrangères et Européennes (Alliance Grant 22836ZJ), the Agence Nationale de la Recherche, and the Conseil Regional de Bourgogne (PARI Photcom).

\section{References}

[1] Y. R. Shen, Principles of Nonlinear Optics, John Wiley \& Sons, New York, NY, USA, 1984.

[2] J. C. Knight, "Photonic crystal fibres," Nature, vol. 424, no. 6950, pp. 847-851, 2003.

[3] B. J. Eggleton, B. Luther-Davies, and K. Richardson, "Chalcogenide photonics," Nature Photonics, vol. 5, no. 3, pp. 141$148,2011$.
[4] G. P. Agrawal, Nonlinear Fiber Optics, Academic Press, San Diego, Calif, USA, 4th edition, 2006.

[5] A. Hasegawa and F. Tappert, "Transmission of stationary nonlinear optical pulses in dispersive dielectric fibers I. Anomalous dispersion," Applied Physics Letters, vol. 23, no. 3, pp. 142-144, 1973.

[6] L. F. Mollenauer and J. P. Gordon, Solitons in Optical Fibers: Fundamentals and Applications, Academic Press, San Diego, Calif, USA, 2006.

[7] W. J. Tomlinson, R. H. Stolen, and A. M. Johnson, "Optical wave breaking of pulses in nonlinear optical fibers," Optics Letters, vol. 10, no. 9, pp. 457-459, 1985.

[8] D. Anderson, M. Desaix, M. Lisak, and M. L. QuirogaTeixeiro, "Wave breaking in nonlinear-optical fibers," Journal of the Optical Society of America B, vol. 9, no. 8, pp. 13581361, 1992.

[9] C. Finot, B. Kibler, L. Provost, and S. Wabnitz, "Beneficial impact of wave-breaking for coherent continuum formation in normally dispersive nonlinear fibers," Journal of the Optical Society of America B, vol. 25, no. 11, pp. 1938-1948, 2008.

[10] J. Azaña, L. K. Oxenløwe, E. Palushani et al., "In-fiber subpicosecond pulse shaping for nonlinear optical telecommunication data processing at $640 \mathrm{Gbit} / \mathrm{s}$," International Journal of Optics, vol. 2012, Article ID 895281, 16 pages, 2012.

[11] A. M. Weiner, "Femtosecond pulse shaping using spatial light modulators," Review of Scientific Instruments, vol. 71, no. 5, pp. 1929-1960, 2000. 
[12] A. M. Clarke, D. G. Williams, M. A. F. Roelens, and B. J. Eggleton, "Reconfigurable optical pulse generator employing a Fourier-domain programmable optical processor," Journal of Lightwave Technology, vol. 28, no. 1, pp. 97-103, 2010.

[13] D. Nguyen, M. U. Piracha, D. Mandridis, and P. J. Delfyett, "Dynamic parabolic pulse generation using temporal shaping of wavelength to time mapped pulses," Optics Express, vol. 19, no. 13, pp. 12305-12311, 2011.

[14] J. Ye, L. S. Yan, W. Pan et al., "Photonic generation of triangular-shaped pulses based on frequency-to-time conversion," Optics Letters, vol. 36, no. 8, pp. 1458-1460, 2011.

[15] P. Petropoulos, M. Ibsen, A. D. Ellis, and D. J. Richardson, "Rectangular pulse generation based on pulse reshaping using a superstructured fiber Bragg grating," Journal of Lightwave Technology, vol. 19, no. 5, pp. 746-752, 2001.

[16] D. Krčmařík, R. Slavík, Y. G. Park, and J. Azaña, "Nonlinear pulse compression of picosecond parabolic-like pulses synthesized with a long period fiber grating filter," Optics Express, vol. 17, no. 9, pp. 7074-7087, 2009.

[17] T. Hirooka, M. Nakazawa, and K. Okamoto, "Bright and dark $40 \mathrm{GHz}$ parabolic pulse generation using a picosecond optical pulse train and an arrayed waveguide grating," Optics Letters, vol. 33, no. 10, pp. 1102-1104, 2008.

[18] K. Kashiwagi, H. Ishizu, and T. Kurokawa, "Fiber transmission characteristics of parabolic pulses generated by optical pulse synthesizer," Japanese Journal of Applied Physics, vol. 50, no. 9, Article ID 092501, 2011.

[19] B. Kibler, R. Fischer, P. A. Lacourt et al., "Optimised one-step compression of femtosecond fibre laser soliton pulses around $1550 \mathrm{~nm}$ to below $30 \mathrm{fs}$ in highly nonlinear fibre," Electronics Letters, vol. 43, no. 17, pp. 915-916, 2007.

[20] A. A. Amorim, M. V. Tognetti, P. Oliveira et al., "Sub-twocycle pulses by soliton self-compression in highly nonlinear photonic crystal fibers," Optics Letters, vol. 34, no. 24, pp. 3851-3853, 2009.

[21] L. F. Mollenauer, R. H. Stolen, and J. P. Gordon, "Experimental observation of picosecond pulse narrowing and solitons in optical fibers," Physical Review Letters, vol. 45, no. 13, pp. 1095-1098, 1980.

[22] S. V. Chernikov and P. V. Mamyshev, "Femtosecond soliton propagation in fibers with slowly decreasing dispersion," Journal of the Optical Society of America B, vol. 8, no. 8, pp. 1633-1641, 1991.

[23] S. V. Chernikov, D. J. Richardson, E. M. Dianov, and D. N. Payne, "Picosecond soliton pulse compressor based on dispersion decreasing fibre," Electronics Letters, vol. 28, no. 19, pp. 1842-1844, 1992.

[24] S. V. Chernikov, J. R. Taylor, and R. Kashyap, "Comblike dispersion-profiled fiber for soliton pulse train generation," Optics Letters, vol. 19, no. 8, pp. 539-541, 1994.

[25] J. Fatome, S. Pitois, and G. Millot, "20-GHz-to-1-THz repetition rate pulse sources based on multiple four-wave mixing in optical fibers," IEEE Journal of Quantum Electronics, vol. 42, no. 10, Article ID 01703695, pp. 1038-1046, 2006.

[26] K. Tai, A. Hasegawa, and A. Tomita, "Observation of modulational instability in optical fibers," Physical Review Letters, vol. 56, no. 2, pp. 135-138, 1986.

[27] B. Ortaç, A. Hideur, G. Martel, and M. Brunel, "2-GHz passive harmonically mode-locked Yb-doped double-clad fiber laser," Applied Physics B, vol. 81, no. 4, pp. 507-509, 2005.

[28] A. Komarov, H. Leblond, and F. Sanchez, "Passive harmonic mode-locking in a fiber laser with nonlinear polarization rotation," Optics Communications, vol. 267, no. 1, pp. 162169, 2006.
[29] A. Komarov, A. Haboucha, and F. Sanchez, "Ultrahigh-repetition-rate bound-soliton harmonic passive mode-locked fiber lasers," Optics Letters, vol. 33, no. 19, pp. 2254-2256, 2008.

[30] J. Schröder, S. Coen, F. Vanholsbeeck, and T. Sylvestre, "Passively mode-locked Raman fiber laser with $100 \mathrm{GHz}$ repetition rate," Optics Letters, vol. 31, no. 23, pp. 3489-3491, 2006.

[31] K. Hammani, B. Kibler, C. Finot et al., "Peregrine soliton generation and breakup in standard telecommunications fiber," Optics Letters, vol. 36, no. 2, pp. 112-114, 2011.

[32] R. Trebino, Frequency-Resolved Optical Gating: The Measurement of Ultrashort Laser Pulses, Kluwer Academic, Norwell, Mass, USA, 2000.

[33] J. Fatome, S. Pitois, C. Fortier et al., "Multiple four-wave mixing in optical fibers: $1.5-3.4-\mathrm{THz}$ femtosecond pulse sources and real-time monitoring of a $20-\mathrm{GHz}$ picosecond source," Optics Communications, vol. 283, no. 11, pp. 24252429, 2010.

[34] S. Pitois, C. Finot, J. Fatome, B. Sinardet, and G. Millot, "Generation of $20-\mathrm{GHz}$ picosecond pulse trains in the normal and anomalous dispersion regimes of optical fibers," Optics Communications, vol. 260, no. 1, pp. 301-306, 2006.

[35] C. Finot, J. Fatome, S. Pitois, and G. Millot, "All-fibered highquality low duty-cycle 20-GHz and 40-GHz picosecond pulse sources," IEEE Photonics Technology Letters, vol. 19, no. 21, pp. 1711-1713, 2007.

[36] C. Fortier, B. Kibler, J. Fatome, C. Finot, S. Pitois, and G. Millot, "All-fibered high-quality low duty-cycle 160-GHz femtosecond pulse source," Laser Physics Letters, vol. 5, no. 11, pp. 817-820, 2008.

[37] I. E. Mansouri, J. Fatome, C. Finot, S. Pitois, and M. Lintz, "All-fibered high-quality stable 20 - and $40-\mathrm{GHz}$ picosecond pulse generators for $160-\mathrm{Gb} / \mathrm{s}$ OTDM applications," IEEE Photonics Technology Letters, vol. 23, no. 20, pp. 1487-1489, 2011.

[38] N. Akhmediev and V. I. Korneev, "Modulation instability and periodic solutions of the nonlinear Schrödinger equation," Theoretical and Mathematical Physics, vol. 69, no. 2, pp. 1089-1093, 1986.

[39] K. Hammani, B. Wetzel, B. Kibler et al., "Spectral dynamics of modulation instability described using Akhmediev breather theory," Optics Letters, vol. 36, no. 11, pp. 2140-2142, 2011.

[40] B. Kibler, J. Fatome, C. Finot et al., "The Peregrine soliton in nonlinear fibre optics," Nature Physics, vol. 6, no. 10, pp. 790-795, 2010.

[41] D. H. Peregrine, "Water waves, nonlinear Schrödinger equations and their solutions," The Journal of the Australian Mathematical Society B, vol. 25, no. 1, pp. 16-43, 1983.

[42] J. M. Dudley, C. Finot, G. Millot et al., "Extreme events in optics: challenges of the MANUREVA project," European Physical Journal, vol. 185, no. 1, pp. 125-133, 2010.

[43] D. R. Solli, C. Ropers, P. Koonath, and B. Jalali, "Optical rogue waves," Nature, vol. 450, no. 7172, pp. 1054-1057, 2007.

[44] J. M. Dudley, C. Finot, G. Millot, and D. J. Richardson, "Selfsimilarity in ultrafast nonlinear optics," Nature Physics, vol. 3, no. 9, pp. 597-603, 2007.

[45] M. E. Fermann, V. I. Kruglov, B. C. Thomsen, J. M. Dudley, and J. D. Harvey, "Self-similar propagation and amplification of parabolic pulses in optical fibers," Physical Review Letters, vol. 84, no. 26, pp. 6010-6013, 2000.

[46] S. Boscolo, S. K. Turitsyn, V. Y. Novokshenov, and J. H. B. Nijhof, "Self-similar parabolic optical solitary waves," 
Theoretical and Mathematical Physics, vol. 133, no. 3, pp. 1647-1656, 2002.

[47] V. L. Kruglov and J. D. Harvey, "Asymptotically exact parabolic solutions of the generalized nonlinear Schrödinger equation with varying parameters," Journal of the Optical Society of America B, vol. 23, no. 12, pp. 2541-2550, 2006.

[48] C. Finot and L. Wu, "Influence of the initial phase profile on the asymptotic self-similar parabolic dynamics," Journal of Nonlinear Optical Physics and Materials, vol. 18, no. 4, pp. 709-721, 2009.

[49] D. Anderson, M. Desaix, M. Karlsson, M. Lisak, and M. L. Quiroka-Teixeiro, "Wave-breaking-free pulses in nonlinearoptical fibers," Journal of the Optical Society of America B, vol. 10, no. 7, pp. 1185-1190, 1993.

[50] C. Finot, G. Millot, and J. M. Dudley, "Asymptotic characteristics of parabolic similariton pulses in optical fiber amplifiers," Optics Letters, vol. 29, no. 21, pp. 2533-2535, 2004.

[51] C. Billet, J. M. Dudley, N. Joly, and J. C. Knight, "Intermediate asymptotic evolution and photonic bandgap fiber compression of optical similaritons around $1550 \mathrm{~nm}$," Optics Express, vol. 13, no. 9, pp. 3236-3241, 2005.

[52] B. G. Bale and S. Boscolo, "Impact of third-order fibre dispersion on the evolution of parabolic optical pulses," Journal of Optics A, vol. 12, no. 1, Article ID 015202, 2010.

[53] B. G. Bale, S. Boscolo, K. Hammani, and C. Finot, "Effects of fourth-order fiber dispersion on ultrashort parabolic optical pulses in the normal dispersion regime," Journal of the Optical Society of America B, vol. 28, no. 9, pp. 2059-2065, 2011.

[54] K. Hammani, C. Finot, S. Pitois, J. Fatome, and G. Millot, "Real-time measurement of long parabolic optical similaritons," Electronics Letters, vol. 44, no. 21, pp. 1239-1240, 2008.

[55] C. Finot, B. Barviau, G. Millot, A. Guryanov, A. Sysoliatin, and S. Wabnitz, "Parabolic pulse generation with active or passive dispersion decreasing optical fibers," Optics Express, vol. 15, no. 24, pp. 15824-15835, 2007.

[56] J. P. Limpert, T. Schreiber, T. Clausnitzer et al., "High-power femtosecond Yb-doped fiber amplifier," Optics Express, vol. 10, no. 14, pp. 628-638, 2002.

[57] A. Malinowski, A. Piper, J. H. V. Price et al., "Ultrashort-pulse $\mathrm{Yb}^{3+}$-fiber-based laser and amplifier system producing $>25$ W average power," Optics Letters, vol. 29, no. 17, pp. 20732075, 2004.

[58] P. Dupriez, C. Finot, A. Malinowski et al., "High-power, high repetition rate picosecond and femtosecond sources based on Yb-doped fiber amplification of VECSELs," Optics Express, vol. 14, no. 21, pp. 9611-9616, 2006.

[59] D. N. Papadopoulos, Y. Zaouter, M. Hanna et al., "Generation of $63 \mathrm{fs} 4.1 \mathrm{MW}$ peak power pulses from a parabolic fiber amplifier operated beyond the gain bandwidth limit," Optics Letters, vol. 32, no. 17, pp. 2520-2522, 2007.

[60] C. Finot, G. Millot, S. Pitois, C. Billet, and J. M. Dudley, "Numerical and experimental study of parabolic pulses generated via Raman amplification in standard optical fibers," IEEE Journal on Selected Topics in Quantum Electronics, vol. 10, no. 5, pp. 1211-1218, 2004.

[61] S. Zhang, C. Jin, Y. Meng, X. Wang, and H. Li, "Propagation of high-power parabolic pulses in cubicon fiber amplifiers," Journal of the Optical Society of America B, vol. 27, no. 6, pp. 1272-1278, 2010.

[62] Vladimir I. Kruglov, Claude Aguergaray, and John D. Harvey, "Propagation and breakup of pulses in fiber amplifiers and dispersion-decreasing fibers with third-order dispersion," Physical Review A, vol. 84, no. 2, Article ID 023823, 14 pages, 2011.
[63] F. Ö. Ilday, J. R. Buckley, W. G. Clark, and F. W. Wise, "Selfsimilar evolution of parabolic pulses in a laser," Physical Review Letters, vol. 92, no. 21, Article ID 213902, 4 pages, 2004.

[64] F. W. Wise, A. Chong, and W. H. Renninger, "High-energy femtosecond fiber lasers based on pulse propagation at normal dispersion," Laser and Photonics Reviews, vol. 2, no. 1-2, pp. 58-73, 2008.

[65] C. Aguergaray, D. Méchin, V. I. Kruglov, and J. D. Harvey, "Experimental realization of a mode-locked parabolic Raman fiber oscillator," Optics Express, vol. 18, no. 8, pp. 8680-8687, 2010.

[66] B. Oktem, C. Ülgüdür, and F. O. Ilday, "Soliton-similariton fibre laser," Nature Photonics, vol. 4, no. 5, pp. 307-311, 2010.

[67] T. Hirooka and M. Nakazawa, "Parabolic pulse generation by use of a dispersion-decreasing fiber with normal groupvelocity dispersion," Optics Letters, vol. 29, no. 5, pp. 498500, 2004.

[68] B. Kibler, C. Billet, P. A. Lacourt, R. Ferriere, L. Larger, and J. M. Dudley, "Parabolic pulse generation in comb-like profiled dispersion decreasing fibre," Electronics Letters, vol. 42, no. 17, pp. 965-966, 2006.

[69] A. Plocky, A. A. Sysoliatin, A. I. Latkin et al., "Experiments on the generation of parabolic pulses in waveguides with lengthvarying normal chromatic dispersion," JETP Letters, vol. 85, pp. 319-322, 2007.

[70] C. Finot, L. Provost, P. Petropoulos, and D. J. Richardson, "Parabolic pulse generation through passive nonlinear pulse reshaping in a normally dispersive two segment fiber device," Optics Express, vol. 15, no. 3, pp. 852-864, 2007.

[71] S. Boscolo, A. I. Latkin, and S. K. Turitsyn, "Passive nonlinear pulse shaping in normally dispersive fiber systems," IEEE Journal of Quantum Electronics, vol. 44, no. 12, pp. 11961203, 2008.

[72] H. Wang, A. I. Latkin, S. Boscolo, P. Harper, and S. K. Turitsyn, "Generation of triangular-shaped optical pulses in normally dispersive fibre," Journal of Optics, vol. 12, no. 3, Article ID 035205, 2010.

[73] S. Boscolo and S. K. Turitsyn, "Pulse shaping in modelocked ring-cavity fibre lasers," in Proceedings of Lasers and Electro-Optics Europe and 12th European Quantum Electronics Conference (CLEO EUROPE/EQEC '11), Munich, German, May 2011, paper EH4.6.

[74] R. R. Alfano, The Supercontinuum Laser Source, Springer, New York, NY, USA, 2006.

[75] J. M. Dudley and J. R. Taylor, Supercontinuum Generation in Optical Fibers, Cambridge University Press, New York, NY, USA, 2010.

[76] L. Boivin and B. C. Collings, "Spectrum slicing of coherent sources in optical communications," Optical Fiber Technology, vol. 7, no. 1, pp. 1-20, 2001.

[77] Y. Takushima and K. Kikuchi, "10-GHz, over 20-channel multiwavelength pulse source by slicing super-continuum spectrum generated in normal-dispersion fiber," IEEE Photonics Technology Letters, vol. 11, no. 3, pp. 322-324, 1999.

[78] Z. Yusoff, P. Petropoulos, K. Furusawa, T. M. Monro, and D. J. Richardson, "A 36-Channel x 10-GHz spectrally sliced pulse source based on supercontinuum generation in normally dispersive highly nonlinear holey fiber," IEEE Photonics Technology Letters, vol. 15, no. 12, pp. 1689-1691, 2003.

[79] F. Parmigiani, C. Finot, K. Mukasa et al., "Ultra-flat SPMbroadened spectra in a highly nonlinear fiber using parabolic pulses formed in a fiber Bragg grating," Optics Express, vol. 14, no. 17, pp. 7617-7622, 2006. 
[80] J. M. Dudley, G. Genty, and S. Coen, "Supercontinuum generation in photonic crystal fiber," Reviews of Modern Physics, vol. 78, no. 4, pp. 1135-1184, 2006.

[81] M. Nakazawa, K. Tamura, H. Kubota, and E. Yoshida, "Coherence degradation in the process of supercontinuum generation in an optical fiber," Optical Fiber Technology, vol. 4, no. 2, pp. 215-223, 1998.

[82] A. M. Heidt, "Pulse preserving flat-top supercontinuum generation in all-normal dispersion photonic crystal fibers," Journal of the Optical Society of America B, vol. 27, no. 3, pp. 550-559, 2010.

[83] L. E. Hooper, P. J. Mosley, A. C. Muir, W. J. Wadsworth, and J. C. Knight, "Coherent supercontinuum generation in photonic crystal fiber with all-normal group velocity dispersion," Optics Express, vol. 19, no. 6, pp. 4902-4907, 2011.

[84] C. Finot, S. Pitois, and G. Millot, "Regenerative $40 \mathrm{Gbit} / \mathrm{s}$ wavelength converter based on similariton generation," Optics Letters, vol. 30, no. 14, pp. 1776-1778, 2005.

[85] S. Boscolo and S. K. Turitsyn, "All-optical signal regeneration by temporal slicing of nonlinearly flattened optical waveform," IEEE Photonics Technology Letters, vol. 17, no. 6, pp. 1235-1237, 2005.

[86] F. Parmigiani, P. Petropoulos, M. Ibsen, and D. J. Richardson, "Pulse retiming based on XPM using parabolic pulses formed in a fiber Bragg grating," IEEE Photonics Technology Letters, vol. 18, no. 7, pp. 829-831, 2006.

[87] S. Boscolo, S. K. Turitsyn, and K. J. Blow, "Time domain all-optical signal processing at a RZ optical receiver," Optics Express, vol. 13, no. 16, pp. 6217-6227, 2005.

[88] I. O. Nasieva, S. Boscolo, and S. K. Turitsyn, "Bit error rate improvement by nonlinear optical decision element," Optics Letters, vol. 31, no. 9, pp. 1205-1207, 2006.

[89] T. T. Ng, F. Parmigiani, M. Ibsen, Z. Zhang, P. Petropoulos, and D. J. Richardson, "Compensation of linear distortions by using XPM with parabolic pulses as a time lens," IEEE Photonics Technology Letters, vol. 20, no. 13, pp. 1097-1099, 2008.

[90] T. Hirooka and M. Nakazawa, "All-optical 40-GHz timedomain Fourier transformation using XPM with a dark parabolic pulse," IEEE Photonics Technology Letters, vol. 20, no. 22, pp. 1869-1871, 2008.

[91] J. Li, B. E. Olsson, M. Karlsson, and P. A. Andrekson, “OTDM add-drop multiplexer based on XPM-induced wavelength shifting in highly nonlinear fiber," Journal of Lightwave Technology, vol. 23, no. 9, pp. 2654-2661, 2005.

[92] F. Parmigiani, P. Petropoulos, M. Ibsen, P. J. Almeida, T. T. $\mathrm{Ng}$, and D. J. Richardson, "Time domain add-drop multiplexing scheme enhanced using a saw-tooth pulse shaper," Optics Express, vol. 17, no. 10, pp. 8362-8369, 2009.

[93] F. Parmigiani, M. Ibsen, T. T. Ng, L. Provost, P. Petropoulos, and D. J. Richardson, "An efficient wavelength converter exploiting a grating-based saw-tooth pulse shaper," IEEE Photonics Technology Letters, vol. 20, no. 17, pp. 1461-1463, 2008.

[94] F. Parmigiani, M. Ibsen, P. Petropoulos, and D. J. Richardson, "Efficient all-optical wavelength-conversion scheme based on a saw-tooth pulse shaper," IEEE Photonics Technology Letters, vol. 21, no. 24, Article ID 5291729, pp. 1837-1839, 2009.

[95] N. Verscheure and C. Finot, "Pulse doubling and wavelength conversion through triangular nonlinear pulse reshaping," Electronics Letters, vol. 47, no. 21, pp. 1194-1196, 2011.

[96] A. I. Latkin, S. Boscolo, R. S. Bhamber, and S. K. Turitsyn, "Doubling of optical signals using triangular pulses," Journal of the Optical Society of America B, vol. 26, no. 8, pp. 14921496, 2009.
[97] R. S. Bhamber, S. Boscolo, A. I. Latkin, and S. K. Turitsyn, "All-optical TDM to WDM signal conversion and partial regeneration using XPM with triangular pulses," in Proceedings of the 34th European Conference on Optical Communication (ECOC '08), pp. 1-2, Brussels, Belgium, 2008, paper Th.1.B.2.

[98] E. R. Andresen, J. M. Dudley, D. Oron, C. Finot, and H. Rigneault, "Transform-limited spectral compression by selfphase modulation of amplitude-shaped pulses with negative chirp," Optics Letters, vol. 36, no. 5, pp. 707-709, 2011.

[99] C. Finot, F. Parmigiani, P. Petropoulos, and D. J. Richardson, "Parabolic pulse evolution in normally dispersive fiber amplifiers preceding the similariton formation regime," Optics Express, vol. 14, no. 8, pp. 3161-3170, 2006.

[100] P. V. Mamyshev, "All-optical data regeneration based on selfphase modulation effect," in Proceedings of the 24th European Conference on Optical Communication (ECOC '98), pp. 475476, Madrid, Spain, September 1998.

[101] L. Provost, C. Finot, P. Petropoulos, K. Mukasa, and D. J. Richardson, "Design scaling rules for 2R-optical self-phase modulation-based regenerators," Optics Express, vol. 15, no. 8, pp. 5100-5113, 2007.

[102] C. Finot, J. Fatome, S. Pitois, G. Millot, and E. Pincemin, "Active Mamyshev regenerator," Optical Review, vol. 18, no. 3, pp. 257-263, 2011.

[103] M. Romagnoli, P. Franco, R. Corsini, A. Schiffini, and M. Midrio, "Time-domain Fourier optics for polarization-mode dispersion compensation," Optics Letters, vol. 24, no. 17, pp. 1197-1199, 1999.

[104] L. A. Jiang, M. E. Grein, H. A. Haus, E. P. Ippen, and H. Yokoyama, "Timing jitter eater for optical pulse trains," Optics Letters, vol. 28, no. 2, pp. 78-80, 2003.

[105] M. Nakazawa and T. Hirooka, "Distortion-free optical transmission using time-domain optical Fourier transformation and transform-limited optical pulses," Journal of the Optical Society of America B, vol. 22, no. 9, pp. 1842-1855, 2005.

[106] T. Hirooka and M. Nakazawa, "Optical adaptive equalization of high-speed signals using time-domain optical Fourier transformation," Journal of Lightwave Technology, vol. 24, no. 7, pp. 2530-2540, 2006.

[107] N. Yoshikane, I. Morita, T. Tsuritani, A. Agata, N. Edagawa, and S. Akiba, "Benefit of SPMbased all-optical reshaper for long-haul DWDM transmission systems," Journal of Selected Topics in Quantum Electronics, vol. 10, pp. 412-420, 2004.

[108] M. Matsuura, N. Kishi, and T. Miki, "Performance improvement of optical RZ-receiver by utilizing an all-optical waveform converter," Optics Express, vol. 13, no. 13, pp. 50745079, 2005.

[109] P. Ghelfi, M. Secondini, M. Scaffardi, F. Fresi, A. Bogoni, and L. Poti, "Impact of an additional all-optical decision element in band-limited receivers for RZ systems," Journal of Lightwave Technology, vol. 25, no. 7, pp. 1728-1734, 2007.

[110] M. Rochette, J. N. Kutz, J. L. Blows, D. Moss, J. T. Mok, and B. J. Eggleton, "Bit-error-ratio improvement with $2 \mathrm{R}$ optical regenerators," IEEE Photonics Technology Letters, vol. 17, no. 4, pp. 908-910, 2005.

[111] M. Rochette, J. L. Blows, and B. J. Eggleton, "3R optical regeneration: an all-optical solution with BER improvement," Optics Express, vol. 14, no. 14, pp. 6414-6427, 2006. 

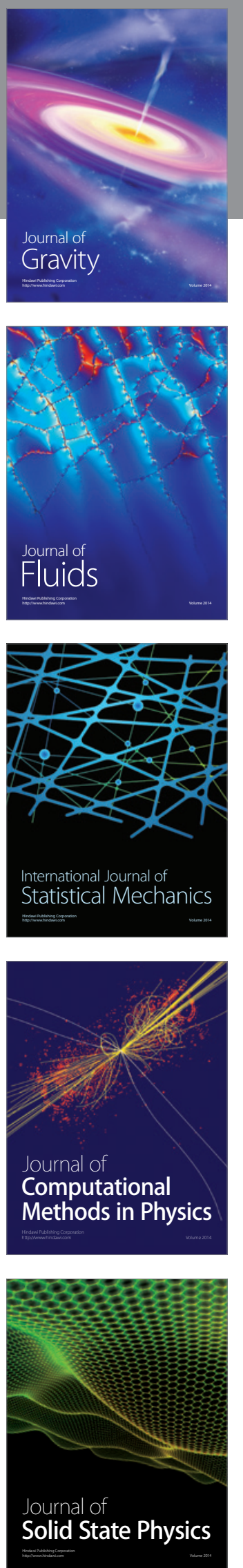

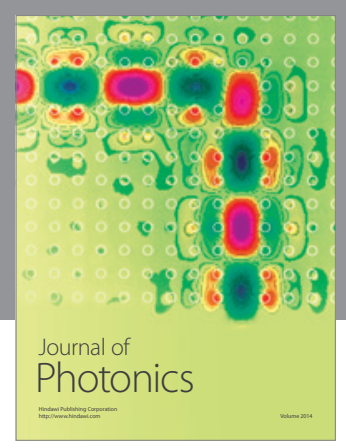

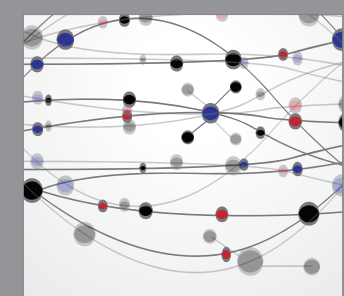

The Scientific World Journal
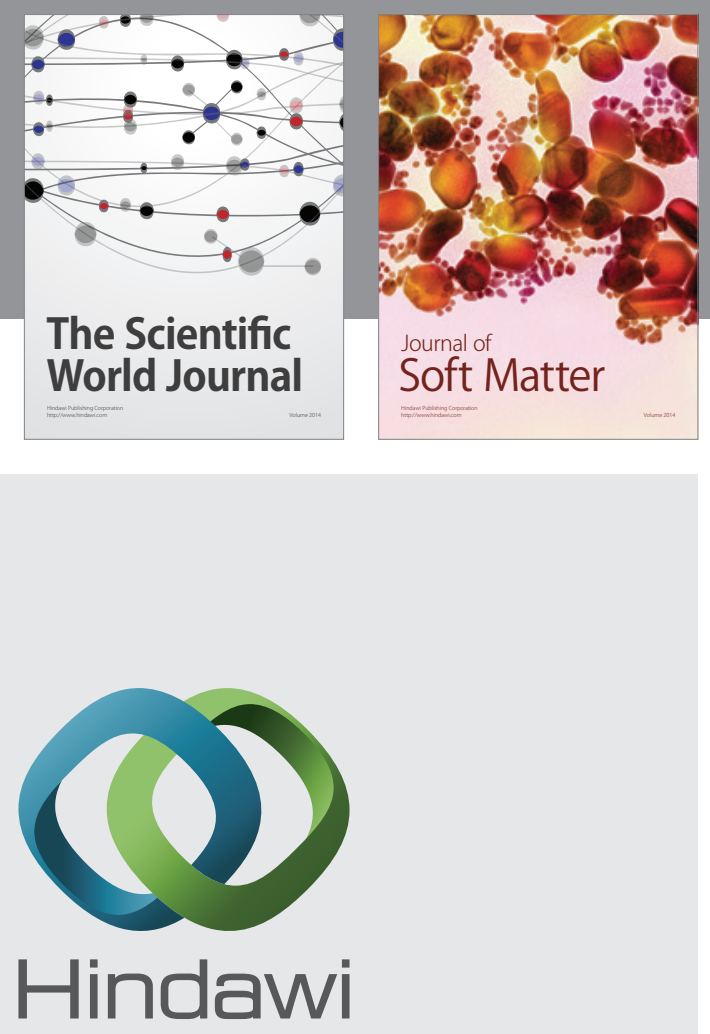

Submit your manuscripts at

http://www.hindawi.com
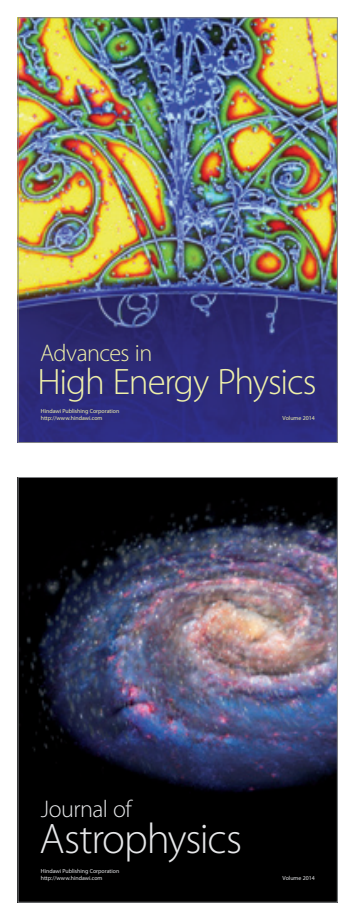
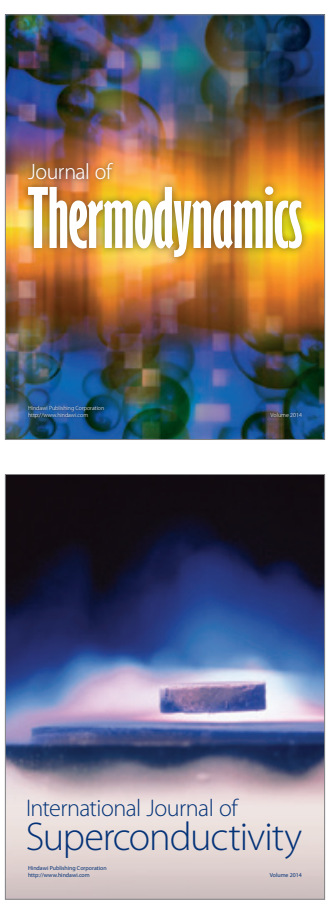
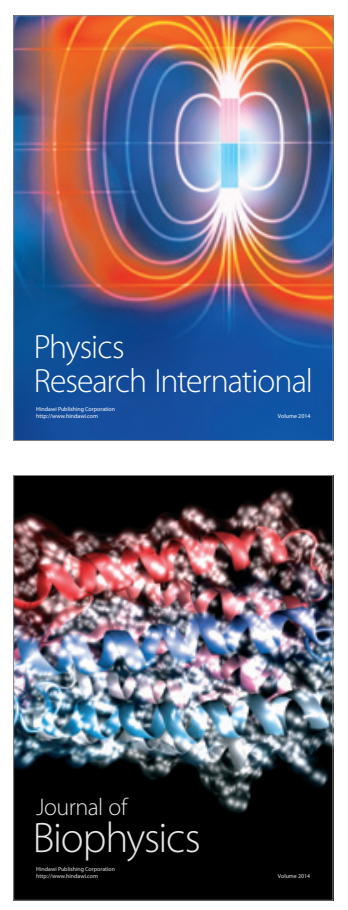
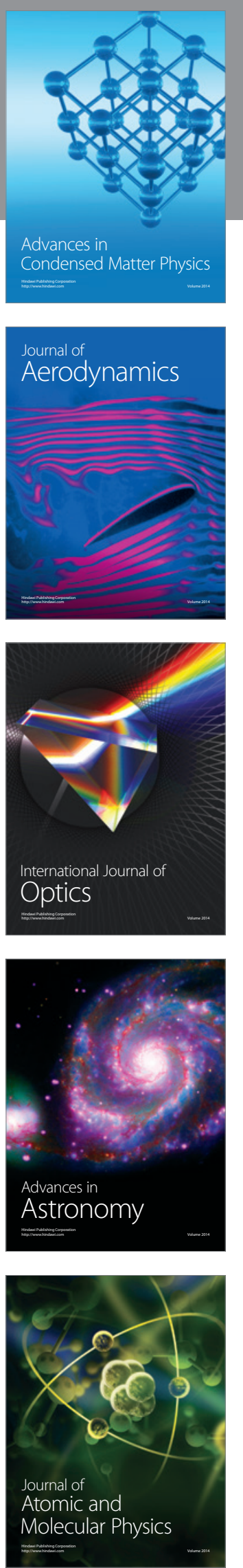